\title{
Stability-Indicating HPLC Method for the Simultaneous Determination of Paracetamol and Tramadol Hydrochloride in Fixed-Dose Combination Tablets
}

\author{
Monica Mame Soma Nyansa ${ }^{1, ~ *, ~ P a t r i c k ~ D o e ~ F i a w o y i f e ~}{ }^{2}$, Nana Ama Mireku-Gyimah ${ }^{3}$, \\ John Nii Adotey Addotey ${ }^{1}$ \\ ${ }^{1}$ Department of Pharmaceutical Chemistry, Faculty of Pharmacy and Pharmaceutical Sciences, Kwame Nkrumah University of Science and \\ Technology, Kumasi, Ghana \\ ${ }^{2}$ Manufacturing Division, Ernest Chemists Limited, Tema, Ghana \\ ${ }^{3}$ Department of Pharmacognosy, Faculty of Pharmacy and Pharmaceutical Sciences, Kwame Nkrumah University of Science and Technology, \\ Kumasi, Ghana
}

\section{Email address:}

msnsomatic@gmail.com (M. M. S. Nyansa)

*Corresponding author

\section{To cite this article:}

Monica Mame Soma Nyansa, Patrick Doe Fiawoyife, Nana Ama Mireku-Gyimah, John Nii Adotey Addotey. Stability-Indicating HPLC Method for the Simultaneous Determination of Paracetamol and Tramadol Hydrochloride in Fixed-Dose Combination Tablets. International Journal of Biomedical Science and Engineering. Vol. 5, No. 4, 2017, pp. 41-47. doi: 10.11648/j.ijbse.20170504.12

Received: October 17, 2017; Accepted: October 31, 2017; Published: November 29, 2017

\begin{abstract}
An accurate, sensitive, precise and isocratic reversed-phase high-performance liquid chromatography (RP-HPLC) method, using a UV detector for analysis of paracetamol and tramadol hydrochloride in combination tablets has been developed and validated. The best separation was achieved on Zorbax SB $\mathrm{C}_{18} 250 \mathrm{~mm} \times 4.6 \mathrm{~mm}, 5-\mu \mathrm{m}$ particle, with $30: 70$ $(\mathrm{v} / \mathrm{v})$ of acetonitrile: $1 \%$ trifluoroacetic acid as the mobile phase, at a flow rate of $1.5 \mathrm{ml} / \mathrm{min}$. The detection wavelength was set at $271 \mathrm{~nm}$. The method was validated according to the International Conference on Harmonization (ICH) guidelines. The response was a linear function of concentration over the range of $325-2112.50 \mathrm{ppm}$ for paracetamol and $37.5-243.8 \mathrm{ppm}$ for tramadol hydrochloride. The correlation coefficient $\left(\mathrm{r}^{2}\right)$ was found to be 0.9998 for paracetamol and 0.9961 for tramadol hydrochloride. The limits of detection and quantitation were found to be $35.80 \mathrm{ppm}$ and $108.49 \mathrm{ppm}$ for paracetamol and 4.14 ppm and $12.54 \mathrm{ppm}$ for tramadol hydrochloride. The drug was subjected to hydrolytic and thermal stress and was found to decompose slightly under both acidic and basic hydrolytic stress conditions and also under thermal stress. The degradation products produced as a result of the hydrolytic and thermal stress did not affect the detection of both paracetamol and tramadol hydrochloride, and the assay could thus be regarded as stability-indicating. The developed method was used to assay a sample of fixed-dose combination tablets and was found to be suitable for application in the analysis of fixed-dose combination tablets of paracetamol and tramadol hydrochloride for quality control purposes.
\end{abstract}

Keywords: RP-HPLC, Paracetamol, Tramadol Hydrochloride, Stability, ICH

\section{Introduction}

The stability of a drug is defined as the ability of the drug to maintain its physical, chemical, biological and microbial properties during its shelf life [1]. The stability of every pharmaceutical product is affected by certain environmental conditions making stability one of the main important quality requirement for pharmaceutical products. The purpose of stability testing according to the International Conference on Harmonization (ICH) guideline is to provide evidence on how the quality of a drug substance or drug product varies with time under the influence of a variety of environmental factors such as temperature, humidity, oxygen and light [2]. 
Different dosage forms of pharmaceutical drugs exist. Notable amongst them is the combination product which according to U. S. FDA includes 'a product comprised of two or more regulated components, that is drug/device, biologic/device, drug/biologic or drug/device/biologic, that's are physically, chemically or otherwise combined or mixed and produced as a single entity' [3]. Fixed-dose combination drugs are known to provide better therapeutic effect, safety, prevent poly-pharmacy and improve compliance [4]. This has led to the increase in combination products used for the management of various medical conditions including painful conditions. One of such combination products is paracetamol/tramadol hydrochloride combination tablets. The development and validation of stability-indicating methods therefore helps to evaluate the drug product in the presence of its degradation products, excipients and other materials that might be present [1].

Paracetamol also known as acetaminophen is chemically known as $N$-(4-Hydroxyphenyl) acetamide (Figure 1). It is a white or almost white crystalline powder that is sparingly soluble in water, freely soluble in alcohol and very slightly soluble in methylene chloride [5]. It is used widely as an analgesic and antipyretic agent but it has no antiinflammatory properties. Although the exact mechanism of its analgesic action has not been found, it is believed to include the inhibition of prostaglandin synthesis, the inhibition of the nitric oxide pathway and the supraspinal activation of descending serotonergic pathways [6]. Paracetamol is better tolerated than Non-Steroidal Antiinflammatory Drugs (NSAIDs) because of its lack of gastric mucosae damage and platelet activation inhibition [7].

Tramadol hydrochloride is a centrally acting opioid analgesic used for the management of moderate to severe painful conditions. It is chemically known as $(1 R S, 2 R S)-2-$ [(Dimethylamino) methyl]-1-(3-methoxyphenyl) cyclohexanol hydrochloride (Figure 2). It is a white or almost white crystalline powder which is freely soluble in water and in methanol and very slightly soluble in acetone [5]. Tramadol hydrochloride is used for the treatment of painful conditions such as neck pain or waist pain. It acts by activating opioid receptors and inhibiting the neuronal reuptake of noradrenaline and serotonin. Although tramadol affects respiration, it is unlikely to produce clinically relevant respiratory depression at therapeutic dosages [6].

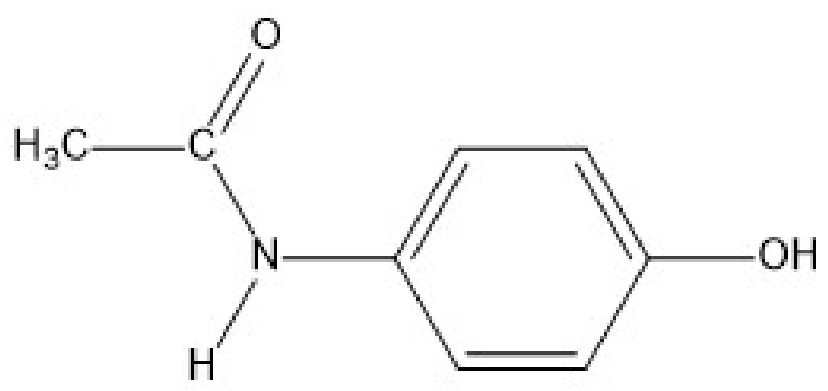

Figure 1. Structure of Paracetamol.

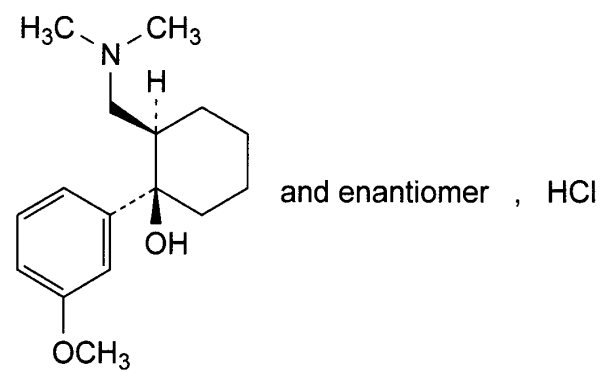

Figure 2. Structure of tramadol hydrochloride.

Tramadol hydrochloride and Paracetamol is an orally administered fixed-dose combination product employed for the symptomatic treatment of moderate to severe pain such as muscoskeletal pain or migraine pain. They provide better analgesia against several types of pain and sources of pain because they have complementary modes of action and target multiple sites [7].

There have been different analytical methods developed and validated for the simultaneous quantification of paracetamol and tramadol hydrochloride with other drug products in fixed-dose combination tablets $[8,9]$. Literature also shows that other methods employing different organic modifiers have been used to quantify both drugs in a simultaneous fashion, in the presence of its degradants. M. V. V. N Murali Krishna et al 2015, developed an Ultra Performance Liquid Chromatography (UPLC) method that simultaneously quantifies acetaminophen and tramadol hydrochloride impurities in combination tablets [10]. Kamble and Singh 2012, described a stability- indicating HPLC method for the analysis of paracetamol and tramadol hydrochloride in a pharmaceutical dosage form employing orthophosphoric acid and methanol as mobile phase [11]. Another study also reported on the stability studies of paracetamol and tramadol hydrochloride in combination with domperidone [12]. This suggests that there are just few HPLC methods that simultaneously analyze paracetamol and tramadol hydrochloride in fixed-dose combination tablets in the presence of its degradation products. Therefore, the objective of this research is to study the degradation behavior of paracetamol and tramadol hydrochloride in fixed-dose combination tablets using different organic modifiers and also to develop and validate an accurate, sensitive and rapid RP-HPLC method for quantifying simultaneously paracetamol and tramadol hydrochloride in fixed-dose combination tablets in the presence of its degradation products.

\section{Experimental}

\subsection{Reagents and Materials}

Pure paracetamol powder (Tianjin Pharmaceutical Co. Ltd., China), pure tramadol hydrochloride (Emmennar Pharma (P) Ltd., India), HPLC grade acetonitrile (Merck, USA), HPLC grade trifluoroacetic acid (Loba Chemie, India), distilled water, paracetamol/ tramadol hydrochloride combination 
tablets (Denk Pharma, Germany).

\subsection{HPLC Instrumentation and Column}

Agilent 1260 Quartenary pump (Germany)

Agilent 1260 Autosampler

Agilent 1260 Variable Wavelength detector

Agilent Openlab software

Zorbax SB $\mathrm{C}_{18}(250 \mathrm{~mm} \times 4.6 \mathrm{~mm})$ column

\subsection{Chromatographic Conditions}

Mobile phase: Acetonitrile: 1\% trifluoroacetic acid (30: 70 $\mathrm{v} / \mathrm{v})$

Flow rate: $1.5 \mathrm{ml} / \mathrm{min}$

Injection volume: $10 \mu \mathrm{l}$

Column temperature: $28^{\circ} \mathrm{C}$

Wavelength: $271 \mathrm{~nm}$

\subsection{Mobile Phase Preparation}

The mobile phase consisted of acetonitrile: $1 \%$ trifluoroacetic acid (30: $70 \mathrm{v} / \mathrm{v})$. The volumes of different components of the mobile phase were measured separately into their individual solvent reservoirs. The mobile phase prepared was filtered through a $0.45 \mu \mathrm{m}$ membrane filter before use.

\subsection{Preparation of Standard Solution}

$325 \mathrm{mg}$ of paracetamol and $37.5 \mathrm{mg}$ of tramadol hydrochloride were accurately weighed and dissolved in 100 $\mathrm{ml}$ of the mobile phase to give a stock solution of $3250 \mathrm{ppm}$ of paracetamol and $375 \mathrm{ppm}$ of tramadol hydrochloride. Furthermore, standard solutions were made by diluting the stock solution with the mobile phase to give solutions in the concentration range of $325-2112.50 \mathrm{ppm}$ for paracetamol and $37.5-243.8 \mathrm{ppm}$ for tramadol hydrochloride.

\subsection{Preparation of Sample Solution}

For sample preparation of the combination tablets, twenty (20) tablets were weighed and finely powdered. A quantity $(0.5000 \mathrm{~g})$ of the powder was weighed and dissolved with $30 \mathrm{ml}$ of the mobile phase. The solution was quantitatively transferred into a $100 \mathrm{ml}$ volumetric flask and sonicated for 5 minutes. It was then made up to the mark and filtered to remove any insoluble excipients. A volume of $5 \mathrm{ml}$ of the solution was pipetted into a $10 \mathrm{ml}$ volumetric flask. The solution was then made to volume using the mobile phase. The solution was injected onto the column three times and the peak areas were recorded.

\subsection{Stress Degradation Studies}

\subsubsection{Acid Hydrolysis}

Acid-induced, forced degradation was performed by adding an aliquot of stock solution of paracetamol and tramadol hydrochloride mixture and $5 \mathrm{M} \mathrm{HCl}$ and refluxing the mixture for approximately six hours. The solution was then left to reach room temperature, neutralized by the addition of the same strength of $\mathrm{HCl}$, and diluted to $100 \mathrm{ml}$ with the mobile phase so as to obtain a final concentration of $162.5 \mathrm{ppm}$ for paracetamol and $18.75 \mathrm{ppm}$ of tramadol hydrochloride.

\subsubsection{Base Hydrolysis}

Base-induced, forced degradation was performed by adding an aliquot of stock solution of paracetamol and tramadol hydrochloride mixture and $5 \mathrm{M} \mathrm{NaOH}$ and refluxing the mixture for approximately six hours. The solution was then left to reach room temperature, neutralized by the addition of the same strength of $\mathrm{NaOH}$, and diluted to $100 \mathrm{ml}$ with the mobile phase so as to obtain a final concentration of $162.5 \mathrm{ppm}$ for paracetamol and $18.75 \mathrm{ppm}$ of tramadol hydrochloride.

\subsubsection{Thermal Degradation}

To study the effect of temperature, approximately 162.5 $\mathrm{mg}$ and $18.75 \mathrm{mg}$ of paracetamol and tramadol hydrochloride mixture was stored at $120^{\circ} \mathrm{C}$ in a controlled temperature oven for 6 hours. It was then dissolved in $100 \mathrm{ml}$ of mobile phase to obtain a solution of final concentration of $1625 \mathrm{ppm}$ and $187.50 \mathrm{ppm}$ of tramadol hydrochloride.

\subsubsection{Stability of Solution}

The solution stability was carried out by storing standard solutions of paracetamol and tramadol hydrochloride mixture in tightly capped volumetric flasks at room temperature for three days. These solutions were assayed after three days against fresh samples.

\subsection{Validation of the Developed Method}

The newly developed method was validated using the various parameters suggested by the International Conference on Harmonization guidelines on validation of analytical procedures: text and methodology. These parameters are linearity, accuracy, specificity, precision and robustness [13].

\section{Results and Discussion}

\subsection{Degradation Behavior of Paracetamol and Tramadol Hydrochloride}

Using HPLC to study the effect of different stress conditions on paracetamol and tramadol hydrochloride, the chromatograms obtained suggest the degradation behavior of both drugs (Table 3). The representative degradation chromatograms are shown in Figures 3a-d. There was major decomposition of the drugs under alkaline hydrolysis, followed by decomposition under acid hydrolysis and thermal degradation (Figures $3 b, 3 c$ and $3 d$ respectively). All these were recorded in a time frame of 5 minutes. 


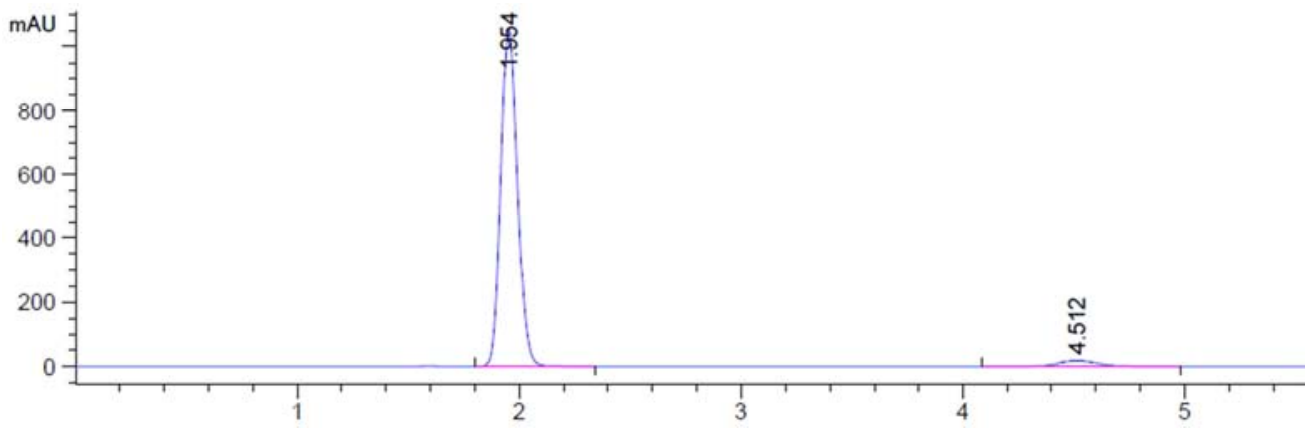

a
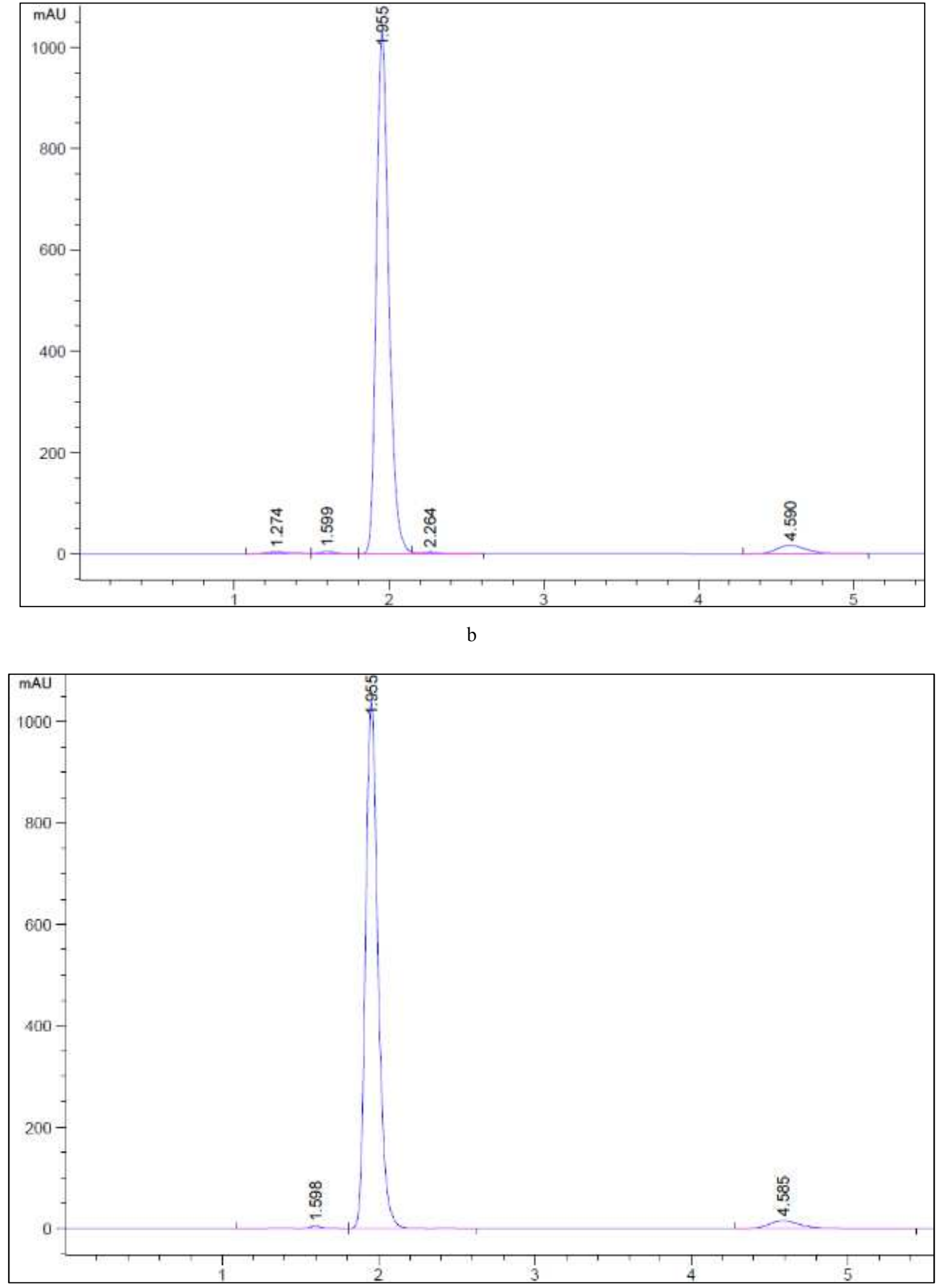


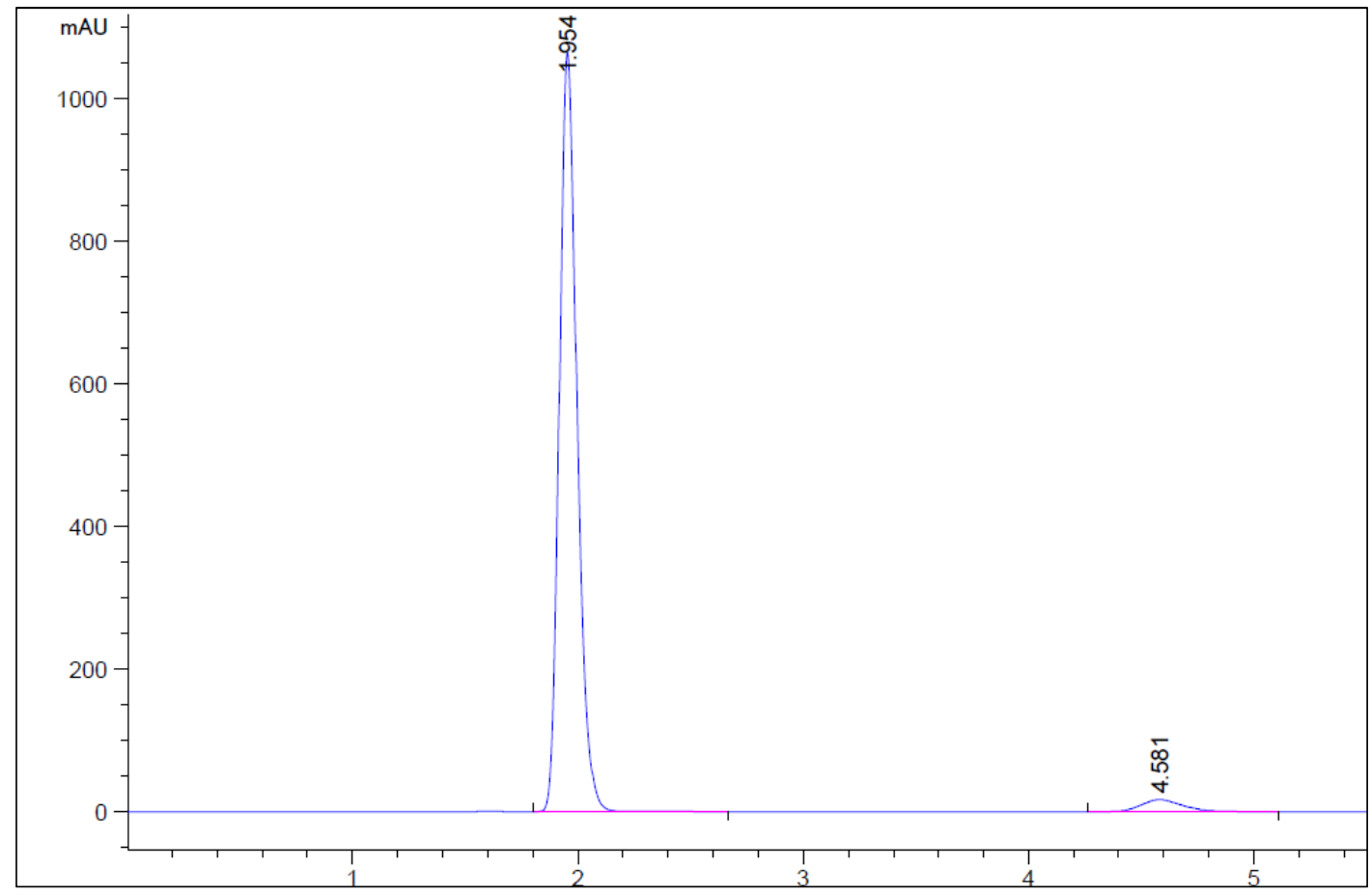

d

Figure 3. Chromatograms ( $x$-axis - time in minutes and y-axis - Absorbance in $m A U$ ) representative of the stability profile of paracetamol and tramadol hydrochloride using this method. (a) Untreated solution (b) Alkaline hydrolysis (c) Acid hydrolysis (d) Thermal hydrolysis.

\subsection{Development of Stability-Indicating Method}

The stability-indicating ability was established under stress conditions. Maximum degradation of the combination product was observed under alkaline conditions, followed by acid hydrolysis and thermal degradation. The percentage degradation of paracetamol and tramadol hydrochloride under stress conditions and retention times of the degradants are shown in Table 1. The separation of paracetamol and tramadol hydrochloride from the degradation products was performed on a Zorbax SB $\mathrm{C}_{18}$ column. Using different proportions of acetonitrile and $1 \%$ trifluoroacetic acid, the drugs were separated to give individual peaks. However, good resolution of the peaks were obtained at a ratio of 30\% of acetonitrile and $70 \%$ of $1 \%$ trifluoroacetic acid $(\mathrm{v} / \mathrm{v})$. Using a variable wavelength UV detector, the eluents were monitored at a wavelength of $271 \mathrm{~nm}$, where maximum sensitivity was observed.

Table 1. Percentage degradation and the retention times of paracetamol and tramadol hydrochloride and their degradants.

\begin{tabular}{llll}
\hline \multirow{2}{*}{ Conditions } & \multirow{2}{*}{ Retention time of drug/degradation products (min) } & \multicolumn{2}{l}{ Percentage degradation of drug } \\
\cline { 3 - 4 } & & Paracetamol & Tramadol hydrochloride \\
\hline Untreated solution & 1.96 & 0.60 & - \\
Acid hydrolysis & $1.60,1.96,4.59$ & 0.50 & 3.26 \\
Alkaline hydrolysis & $1.27,1.60,1.96,2.26,4.59$ & 5.45 & 0.94 \\
Thermal stability & $1.95,4.58$ & & \\
\hline
\end{tabular}

\subsection{Validation of the Developed Method}

The stability-indicating method was validated per the ICH parameters such as specificity, accuracy, linearity, range, precision and robustness [13].

The Limit of Detection (LOD) and Limit of Quantification (LOQ) concentrations for paracetamol and tramadol hydrochloride were found to be $35.80 \mathrm{ppm}$ and $4.14 \mathrm{ppm}$ and $108.49 \mathrm{ppm}$ and $12.54 \mathrm{ppm}$ respectively.

A summary of the method validation results is shown in Table 2 .

Table 2. Summary of method validation parameters for paracetamol and tramadol hydrochloride.

\begin{tabular}{lll}
\hline Parameter & Paracetamol & Tramadol hydrochloride \\
\hline Specificity & Specific & Specific \\
Limit of Detection $(\mathrm{ppm})$ & 35.80 & 4.14 \\
Limit of Quantification $(\mathrm{ppm})$ & 108.49 & 12.54 \\
Linearity $\left(\mathrm{r}^{2}\right)$ & 0.9998 & 0.9961 \\
Robustness & Robust & Robust \\
\hline
\end{tabular}


The system suitability results are shown in Table 3 .

Table 3. System suitability results.

\begin{tabular}{lll}
\hline Description & Paracetamol & Tramadol hydrochloride \\
\hline Peak symmetry & 0.79 & 0.79 \\
Width & 0.0798 & 0.1744 \\
Theoretical plates & 3232 & 3643 \\
Resolution & 2.12 & 11.30 \\
Selectivity & 1.20 & 2.25 \\
\hline
\end{tabular}

The precision and recovery data are listed in Table 4.

Table 4. Precision and recovery data.

\begin{tabular}{lllll}
\hline \multirow{2}{*}{ Samples } & \multirow{2}{*}{ Actual concentration (ppm) } & Precision (\% RSD) & Accuracy \\
\cline { 3 - 5 } & 1300 & Intra-day & Inter-day & 100.70 \\
Paracetamol & 1625 & 0.10 & 0.41 & 100.01 \\
& 1950 & 0.05 & 0.44 & 99.64 \\
\multirow{2}{*}{ Tramadol hydrochloride } & 150 & 0.27 & 0.84 & 97.93 \\
& 187.5 & 0.06 & 0.88 & 98.67 \\
\hline
\end{tabular}

Comparing this method to already existing methods, this method is relatively faster since it requires an analysis time of about 5 minutes as evidenced in the chromatograms (Figure 3).

The influence of small changes in chromatographic conditions on the method was studied. Change in flow rate $(1.5 \mathrm{ml} / \mathrm{min}$ to $2.0 \mathrm{ml} / \mathrm{min})$, column temperature $\left(28^{\circ} \mathrm{C}\right.$ to $30^{\circ} \mathrm{C}$ ) and column (Zorbax $\mathrm{C}_{18}$ to Agilent Prep $\mathrm{C}_{18}$ ) was studied to determine the robustness of the developed method. The $\%$ RSD was found to be $<2 \%$. This suggests that the method is robust and small changes in chromatographic conditions would not affect the assay. The stability of the drug solution was determined by storing it for three days. There were no significant changes over the observation period. This data confirms the stability of the solution for at least three days.

The system suitability testing evaluates the effectiveness of the entire chromatographic system prior to use and during the time of analysis. Parameters such as theoretical plates, resolution and selectivity were all within the limits stipulated by CDER guidelines [14]. The chromatographic system used, can therefore be said to be suitable.

\section{Conclusion}

A simple, accurate and sensitive reversed-phase HPLC method for the simultaneous quantification of paracetamol and tramadol hydrochloride in fixed-dose combination tablets have been developed and validated per the International Conference on Harmonization guidelines. The method was also used to study the stability profile of paracetamol and tramadol hydrochloride under stress conditions. The newly developed method is easy to operate, requires a relatively shorter analysis time and is stability-indicating (it can be used to study the stability profile of paracetamol and tramadol hydrochloride). This new method can therefore be used for the routine analysis of fixed-dose combination tablets of paracetamol and tramadol hydrochloride.

\section{Acknowledgements}

The authors would like to acknowledge Ernest Chemists Limited, Manufacturing Division for their technical assistance.

\section{References}

[1] P. Hamrapurkar, P. Patil, M. Desai, M. Phale, S. Pawar, "Stress degradation studies and development of a validated stability-indicating-assay-method for determination of diacerein in presence of degradation products," Pharm Methods, vol. 2 (1), pp. 30-35, Jan 2011.

[2] ICH Harmonised Tripartite Guideline (2003). Stability Testing of New Drug Substances and Products (Q1AR2).

[3] U. S. Food and Drug Administration (2017). Combination Products.

[4] S. Bangalore, G. Kamalakkannan, S. Parkar and F. H. Messerli, "Fixed-Dose combinations improve medication compliance: A Meta-Analysis,” Am J Med, vol. 120 (8), pp. 713-719, August 2007.

[5] British Pharmacopeia Commission, The British Pharmacopoeia 2013. London: The Stationery Office.

[6] S. Dhillon, "Tramadol/Paracetamol Fixed-Dose Combination; A review of its use in the management of moderate to severe pain," Clin Drug Investig, vol. 30 (10), pp. 711-738, 2010.

[7] S. S. Reuben, "Perioperative use of COX-2 agents," in Current Therapy in Pain, 1st edn. H. Smith, Eds. Philadelphia: Saunders Elsevier, 2009, pp. 60-62.

[8] F. Ghorbani-Bidkorbeh, S. Shakrokhian, A. Mohammadi, R. Dinavard, "Simultaneous voltammetric determination of tramadol and acetaminophen using carbon nanoparticles modified glassy electrode," Electrochimica Acta, vol. 55 (8), pp. 2752-2759, March 2010. 
[9] D. Ravisankar, V. Sridevi, P. G. Kumari, "Development and validation of HPLC method for the simultaneous quantification of acetaminophen and tramadol in combined pharmaceutical dosage forms with UV detection," International Journal of Applied Biology and Pharmaceutical Technology, vol. 6 (2), pp. 174-181, April 2015.

[10] M. V. V. N. M. Krishna, S. V. Rao, N. V. S. Venugopal, B. P. V. Mantena, "Simultaneous determination of acetaminophen and tramadol impurities in combination product of acetaminophen and tramadol tablets by UPLC with trifunctional octadecyl column," TACL, vol. 5, pp. 306-318, 2015.

[11] R. M. Kamble, S. Singh, "Stability-indicating RP-HPLC method for analysis of paracetamol and tramadol in a pharmaceutical dosage form," E-Journal of Chemistry, vol. 9 (3), 1347-1356, 2012.

[12] K. Karunakaran, G. Navaneethan, K. P. Elango, "Development and validation of a stability-indicating RPHPLC method for simultaneous determination of paracetamol, tramadol $\mathrm{HCl}$ and domperidone in a combined dosage form," Trop J Pharm Res, vol. 11 (1), pp. 99-106, March 2012.

[13] ICH Harmonised Tripartite Guideline (1996). Validation of Analytical Procedures: Text and Methodology Q2 (R1).

[14] A. Bose, 'HPLC calibration process parameters in terms of system suitability test," Austin chromatogr, vol. 1 (2), pp. 4, 2014. 